\title{
Title: Does education explain the terminal decline in the oldest-old? Evidence from two longitudinal studies of ageing: Newcastle 85+, UK and OCTO-Twin, Sweden
}

Dorina Cadar PhD1, Blossom C.M. Stephan PhD², Carol Jagger PhD², Boo Johansson PhD 3 , Scott M. Hofer PhD ${ }^{4}$, Andrea M. Piccinin PhD ${ }^{4}$, Graciela Muniz-Terrera PhD ${ }^{1}$

${ }^{1}$ MRC Unit for Lifelong Health and Ageing at University College London, UK; ${ }^{2}$ Psychology Department, Newcastle University, UK; ${ }^{3}$ Psychology Department, University of Gothenburg, Sweden; ${ }^{4}$ Psychology Department, University of Victoria, Canada

\section{Background}

Cognitive performance shows a marked deterioration in close proximity to death, as postulated by terminal decline hypothesis. However, the association between education and terminal decline remains highly controversial. This study investigated the role of education on terminal decline in two European longitudinal studies of oldest-old.

\section{Method}

Participants were from the Newcastle 85+, UK ( $N=702)$ and from OCTO-Twin, Sweden $(\mathrm{N}=845)$. They were assessed biannually over 3 and 5 consecutive waves respectively. In a coordinated analysis, multilevel models were employed to examine the association between education and terminal decline on Mini-Mental State Examination (MMSE), controlling for age at baseline, dementia incidence, sex, and time to death from the study entry within each cohort. Cognitive decline was modeled as a linear function of time to death in both cohorts and as a quadratic function in the OCTO-Twin study (due to longer follow-up). Education was a continuous measure (ranging 6-20yrs in Newcastle 85+ and 0-23yrs in OCTO-Twin).

\section{Results}

The results suggest that a typical British man, aged 85 at baseline, with 10 years education, entered the terminal phase at around 2.5 years before death, and the rate of decline was $-1.04(0.25)$, $p<0.001$ with each year closer to the time of death. In contrast, a Swedish man, aged 83, with an average of 7 years education, entered the study at around 8 years from death, after which the rate of cognitive decline steepened by $-1.70(\mathrm{SE}=0.20), p<0.001$ and accelerated by $-0.11(0.01) p<0.001$ points per year closer to the time of death. Education was positively associated with the estimated MMSE scores prior to death only in OCTO-Twin $(0.43(\mathrm{SE}=0.15) \mathrm{p}=0.003)$ and did not attenuate the rate of terminal decline in either cohort.

\section{Conclusions}

Decline and acceleration of this decline were detectable in both studies prior to death, with steeper rates of decline observed in the Swedish cohort. However, this process was not lessened by education itself. This work contributes to a better understanding of the transition from the subtle cognitive changes accompanying age decline to those of neurological substance, and the role of education on this decline.

\section{Word count: 349 words}


DC has run the analyses and drafted the manuscript. AP and GMT have contributed to the analyses. All authors have contributed to the revision of the manuscript.

The funding sources of this work are the Alzheimer's Society [Grant number 144] and the Medical Research Council [Unit Programme number MC_UU_12019/1]. The authors of this work have no competing interests.

\section{Corresponding author: Dorina Cadar}

d.cadar@ucl.ac.uk

+44 (0)207670 5718 (phone)

+44 (0)2075801501 (fax)

MRC Unit for Lifelong Health and Ageing, London,

33 Bedford Place, WC1B 5JU, UK, London 